\title{
Hemorheological Alterations in Patients with Heart Failure with Reduced Ejection Fraction Treated by Resveratrol
}

\author{
Roland Gal (D), ${ }^{1,2}$ Dora Praksch ${ }^{1},{ }^{1,2}$ Peter Kenyeres, ${ }^{1,2}$ Miklos Rabai, ${ }^{1,2}$ Kalman Toth, ${ }^{1,2}$ \\ Robert Halmosi, ${ }^{1,2}$ and Tamas Habon iD ${ }^{1,2}$ \\ ${ }^{1}$ Division of Cardiology, 1st Department of Medicine, Medical School, University of Pécs, Hungary \\ ${ }^{2}$ Szentágothai Research Centre, University of Pécs, Hungary \\ Correspondence should be addressed to Roland Gal; gal.roland@pte.hu
}

Received 29 February 2020; Accepted 30 May 2020; Published 1 July 2020

Academic Editor: Leonardo De Luca

Copyright (c) 2020 Roland Gal et al. This is an open access article distributed under the Creative Commons Attribution License, which permits unrestricted use, distribution, and reproduction in any medium, provided the original work is properly cited.

\begin{abstract}
Objectives. Several beneficial effects of resveratrol have already been published. This study evaluated the effect of resveratrol on the hemorheological parameters in patients with heart failure with reduced ejection fraction. Methods. In our double-blind, placebocontrolled human clinical trial, we enrolled 60 outpatients with heart failure. Patients were randomized into two groups: receiving either $100 \mathrm{mg}$ resveratrol capsule daily or placebo for 3 months. Hematocrit was determined by microhematocrit centrifuge. Plasma and whole blood viscosity was evaluated by capillary viscometer. Erythrocyte aggregation was measured by both LORCA and Myrenne aggregometers. LORCA ektacytometer was used for measuring erythrocyte deformability. Exercise capacity was assessed by a 6-minute walk test. Results. Resveratrol treatment did not have any significant effect on hematocrit and viscosity. The erythrocyte deformability also remained unchanged. However, significant improvement of red blood cell aggregation was observed in the resveratrol group compared to baseline after 3 months. Furthermore, positive correlation was found between the exercise capacity and the hemorheological properties (Hct, WBV, and RBC aggregation and deformability) as well. Conclusion. These findings indicate that resveratrol can significantly reduce red blood cell aggregation, which may positively influence microcirculation, which may contribute to the improvement of tissue perfusion and oxygen supply in heart failure.
\end{abstract}

\section{Introduction}

Heart failure (HF) continues to be a significant cause of cardiovascular mortality. Over the past few decades, numerous medical and device-based therapies have been developed for the management of heart failure; however, mortality remains high even in optimally treated patients [1]. Heart failure is a systemic, multifactorial disease, in which complex structural, neurohumoral, cellular, and molecular changes lead to volume overload, increased sympathetic activity, and redistribution of circulation and result in different, developing clinical signs and symptoms in parallel $[2,3]$. Complex impairment of peripheral and coronary blood flow in HF including restricted microcirculation, attenuated regulatory mechanisms, and impaired hemorheological properties causes reduced oxygen utilization contributing to the symptoms and progression of heart failure [4-6].
Red blood cell (RBC) aggregation and deformability have an important role in capillary blood flow including coronary microcirculation. Besides many clinical states (e.g., ischemic heart disease, diabetes, and venous thrombosis), heart failure is known to be associated with increased RBC aggregation, which has a negative influence on the in vivo flow dynamics of blood. The reduction of RBC aggregation may have a positive effect on the flow properties of blood, which can be beneficial in cardiovascular diseases [7-10]. Furthermore, when the vascular autoregulatory reserve is exhausted in heart failure, the hemorheological disturbances-which can be easily compensated in healthy individuals-will have deleterious effects. Moreover, rheological disorders were found to be present even in the early stage of cardiovascular diseases, before their massive functional manifestation $[5,6,11]$.

Over the past several decades, numerous reports have demonstrated enhanced expression of inflammatory cytokines 


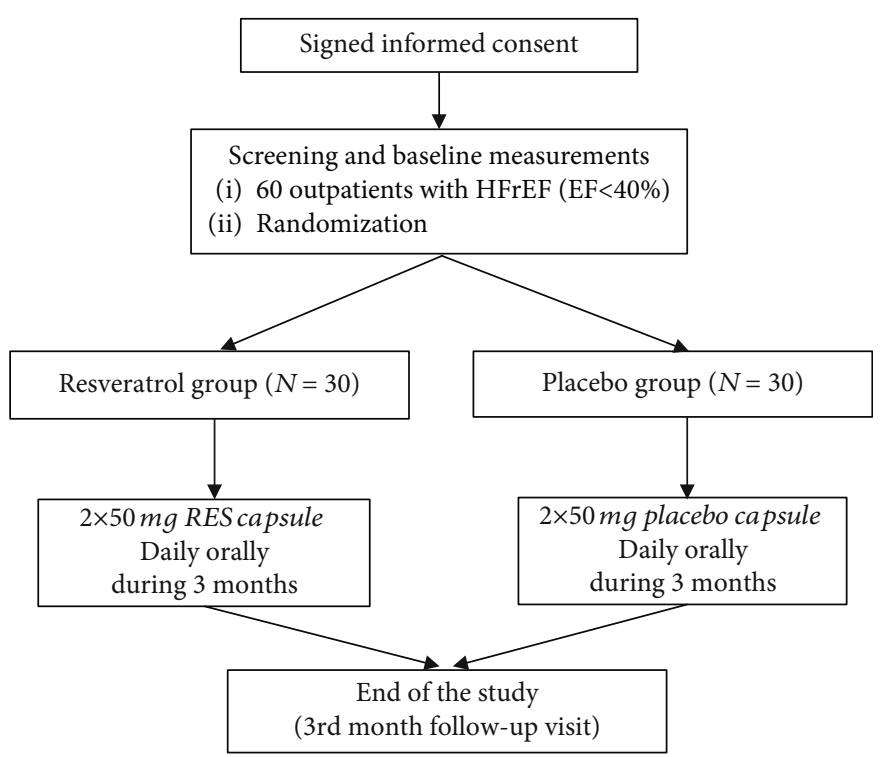

FIgURe 1: Study design.

(e.g., TNF- $\alpha$, IL-1, IL-6, and IL-18) and reactive oxygen species (ROS) in chronic heart failure. These pathological conditions contribute to cardiac damage (remodelling, hypertrophy, fibrosis, etc.) and peripheral vascular disturbances [12-14]. Red blood cells are also prone to oxidative stress being the first cells in the body to be exposed to stressful stimuli, which results in abnormalities in the function, morphology, and metabolism of erythrocytes [15]. The aggregation and deformability of RBCs depend on the cellular properties as well as the mechanical and physicochemical characteristics of their environment (e.g., fibrinogen, large molecular weight globulins) [16], and both are influenced by the aforementioned processes.

French people tend to have a lower incidence of cardiovascular diseases despite having similar coronary risk factors as people in other industrialized countries. This phenomenon is known as the French paradox and is attributed to the higher red wine intake by the French [17].

Red wine contains high amount of polyphenolic compounds like resveratrol (RES), catechin, and quercetin, and RES is considered to be primarily responsible for the cardioprotective effect of red wine. Resveratrol (3,5,4-trihydroxystilbene) is a nonflavonoid polyphenolic compound produced by plants in response to environmental stress. Several mechanisms may be responsible for the cardioprotective effect of RES including reduction of oxidative stress, inflammation, and pathologic hypertrophic signaling and improved $\mathrm{Ca}^{2+}$ handling [18-22]. As these are important factors also in the pathogenesis of heart failure, we supposed that resveratrol may also have protective effects in heart failure.

The effects of moderate red wine consumption and the effectiveness of RES treatment on hemorheological parameters have already been evaluated in some previous animal and human trials. In a previous in vivo study of our working group, moderate red wine consumption had beneficial effect on red blood cell aggregation and deformability in healthy volunteers. Moreover, a significant improvement of RBC deformability and platelet aggregation was observed after RES administration ( $10 \mathrm{mg}$ daily for 3 months) in patients with chronic ischemic heart disease $[8,23,24]$.

According to literature, no effects of RES on rheological properties have been reported in heart failure. Therefore, the aim of this study was to test our hypothesis that resveratrol can improve the hemorheological parameters in patients with heart failure with reduced ejection fraction.

\section{Materials and Methods}

2.1. Study Design. Our trial was a single-center, double-blind, randomized placebo-controlled human study. The study design is summarized in Figure 1. The study was conducted in accordance with the principles stated in the Declaration of Helsinki (1996) and International Conference on Harmonization Good Clinical Practice, as well as local and national regulations. The protocol of the trial was approved by the Regional Ethics Committee of the University of Pécs (license number 5830). Written informed consent has been provided by all patients prior to any study-related procedures.

2.2. Eligibility. 60 stable outpatients (age: $66.7 \pm 2.01$ years, 17 women and 43 men) with heart failure with reduced ejection fraction (HFrEF) in NYHA (New York Heart Association) class II or III were enrolled between $01 / 03 / 2016$ and 30/11/2017 into our study (ejection fraction $(\mathrm{EF})<40 \%$; ischemic/nonischemic: $34 / 26)$. They were randomized into two groups (resveratrol group and placebo group): $100 \mathrm{mg}$ resveratrol capsules were administered orally for 3 months $(2 \times 50 \mathrm{mg})$ in the first group $(n=30)$ and identical placebo capsules in the second group $(n=30)$. The baseline values of RES and placebo groups were compared to the age-matched control group (mean age: $67.15 \pm 1.01$ years, female/male: $11 / 9$ ), without 
heart failure (ejection fraction $>50 \%$ ), and with moderate cardiovascular risk profile.

The resveratrol capsule and the matching placebo were purchased from ARGINA Nutraceuticals Ltd. (Fót, Hungary). The resveratrol capsule is commercially available and has official license for being marketed.

The main exclusion criteria were acute cardiovascular or cerebrovascular event, major cardiac surgery or intervention within 30 days prior to randomization, renal failure (estimated glomerular filtration rate $($ eGFR) $<20 \mathrm{ml} / 1.73$ $\mathrm{m}^{2} / \mathrm{min}$ ), or hepatic impairment (alanine aminotransferase (ALT) or aspartate aminotransferase $(\mathrm{AST}) \geq 2 \mathrm{x}$ upper limit of normal (ULN) at baseline).

All of the involved patients received the evidence-based drug treatment for heart failure with reduced ejection fraction (HFrEF), including angiotensin-converting enzyme (ACE) inhibitors (or angiotensin receptor blocker (ARB)), beta-blockers, mineralocorticoid receptor antagonists (MRA), and in certain cases ivabradine. No patients were on angiotensin receptor-neprilysin inhibitor (ARNI) therapy. The preventive drug regime and the used doses were based on the actual ESC (European Society of Cardiology) heart failure guideline [2].

The patients had baseline and 3-month follow-up visits. During visits, the compliance of the patients was checked according to self-report and counting the remaining capsules at the final (3-month follow-up) visit. During the whole study period, subjects were in stable clinical condition and received unchanged medical therapy (Table 1).

The baseline characteristics and the homogeneity of our randomized population are described in Table 1 . There were no significant differences in epidemiological characteristics between the placebo- and RES-treated groups at baseline.

2.3. Hemorheological Parameters. In this hemorheological substudy, blood samples were taken on the day of randomization and after 3 months from the antecubital vein after a 12-hour fasting. Blood samples for hemorheological measurements were collected into $2 \times 6 \mathrm{ml}$ ethylenediaminetetraacetic acid- (EDTA-) coated Vacutainer tubes with a 21-gauge butterfly infusion set. Hemorheological measurements were carried out within 2 hours after blood sampling [25].

The following hemorheological parameters were determined: hematocrit, plasma and whole blood viscosity, and red blood cell deformability and aggregation.

2.3.1. Hematocrit. Hematocrit (Hct) was measured by Haemofuge microhematocrit centrifuge (Heraeus Instr.; Germany). Measurements were performed at room temperature $\left(22 \pm 1^{\circ} \mathrm{C}\right)$ [26].

2.3.2. Plasma and Whole Blood Viscosity. Plasma viscosity (PV) and whole blood viscosity (WBV) values were determined by a Hevimet 40 capillary viscometer (Hemorex Ltd., Hungary). Plasma was collected after blood sample centrifugation for 10 minutes at $1500 \mathrm{~g}$. Apparent WBV values interpolated to $90 \mathrm{~s}^{-1}$ shear rate were reported. Measurements were performed at $37^{\circ} \mathrm{C}$ [26].
2.3.3. Red Blood Cell Aggregation. Red blood cell aggregation measurements were carried out with a LORCA aggregometer (Laser-assisted Optical Rotational Cell Analyzer; R\&R Mechatronics, Hoorn, The Netherlands) based on syllectometry. $1 \mathrm{ml}$ of oxygenated blood is injected into the gap between a static bob and a rotating cylinder that creates a simple shear flow. Erythrocytes are first disaggregated at high shear rate $\left(500 \mathrm{~s}^{-1}\right)$ then the shearing is stopped. The intensity of backscattered laser light is plotted against time on a syllectogram. The aggregation behavior of blood sample is characterized by the aggregation index (AI) calculated from the first 10 seconds of the syllectogram after the shape recovery period and by the time that elapses until intensity is reduced to half of the peak amplitude $\left(t_{1 / 2}\right)$. The smallest shear rate required for complete disaggregation $(\gamma$ : gamma $(1 / \mathrm{s}))$ was also determined [27].

RBC aggregation was measured also by Myrenne aggregometer (MA-1 Aggregometer, Myrenne Ltd., Germany), applying the light transmission method of SchmidSchönbein et al. This method calculates the aggregation index according to the change in intensity of transmitted infrared light during aggregation either at zero shear $\left(M_{0}\right)$ or low shear $\left(M_{1}\right.$ at $\left.3 \mathrm{~s}^{-1}\right)$ after disaggregation [28].

2.3.4. Red Blood Cell Deformability. For the deformability measurement with LORCA ektacytometer, $25 \mu \mathrm{l}$ blood was suspended in high viscosity $(32.6 \mathrm{mPas})$ polyvinylpyrrolidone solution. RBCs were sheared by shear stress from $0.3 \mathrm{~Pa}$ to $30 \mathrm{~Pa}$, and their deformation was visualized by laser diffraction. The isointensive points of the diffraction pattern draw an ellipse with a longer $A$ and a shorter $B$ diameter. Deformation is characterized by the elongation index calculated by $(A-B) /(A+B)$. Measurements were performed at $37^{\circ} \mathrm{C}$. The deformability results were also analyzed by means of the Lineweaver-Burke nonlinear equation, with calculation of the maximal elongation index $\left(\mathrm{EI}_{\max }\right)$ at theoretical infinite shear and the shear stress value required for half of this maximal elongation $\left(\mathrm{SS}_{1 / 2}\right)$ [29]. However, since goodness-of-fit results proved to be below the acceptable level in several cases and rejecting such cases would have biased results, we only used the raw elongation index (EI) parameters rather than $\mathrm{EI}_{\max }$ and $\mathrm{SS}_{1 / 2}$ in the final analysis.

2.4. Six-Minute Walk Test. The six-minute walk test (6MWT) is a submaximal exercise test that measures the walking distance for 6 minutes. The test was performed on a $30 \mathrm{~m}$ long section of the corridor in our department according to the guideline of American Thoracic Society. The patients were in rest comfortably for 10 minutes prior to the test. The supervisor was a study nurse, who was blinded to the protocol. After 6 minutes or if the patient could not walk any further, the test was stopped and the distance and the reason for stopping (dyspnea, fatigue, chest pain, etc.) were recorded [30].

2.5. Statistical Analysis. SPSS statistical software, version 25, was used for statistical analysis. After using the KolmogorovSmirnov test to check the normality of the data distribution, differences between baseline and 3-month values were analyzed 
TABLE 1: Baseline characteristics of the study population according to the treatment arms.

\begin{tabular}{|c|c|c|}
\hline & $\begin{array}{l}\text { Resveratrol } \\
\quad(n=30)\end{array}$ & $\begin{array}{l}\text { Placebo } \\
(n=30)\end{array}$ \\
\hline Age (year) & $65.8 \pm 1.9$ & $67.5 \pm 2.1$ \\
\hline Male & $22(73 \%)$ & $21(70 \%)$ \\
\hline Ejection fraction (\%) & $30.06 \pm 1.04$ & $31.70 \pm 1.27$ \\
\hline NT-proBNP (pg/ml) & $2998 \pm 507$ & $3139 \pm 446$ \\
\hline Serum creatinine $(\mu \mathrm{mol} / \mathrm{l})$ & $99.77 \pm 4.42$ & $104.47 \pm 4.82$ \\
\hline Sys. BP (mmHg) & $132.47 \pm 3.4$ & $128.77 \pm 3.8$ \\
\hline Dias. BP (mmHg) & $79.1 \pm 2.33$ & $80.13 \pm 2.66$ \\
\hline Heart rate (beat/min) & $72.2 \pm 2.75$ & $76.93 \pm 2.5$ \\
\hline \multicolumn{3}{|l|}{ Etiological factors } \\
\hline Ischemic heart disease & $17(56.7 \%)$ & $17(56.7 \%)$ \\
\hline Nonischemic (alcohol, chemotherapy, and myocarditis) & $13(43.3 \%)$ & $13(43.3 \%)$ \\
\hline \multicolumn{3}{|l|}{ Risk factors, comorbidities } \\
\hline Hypertension & $22(73 \%)$ & $23(76 \%)$ \\
\hline Diabetes & $13(43 \%)$ & $14(46 \%)$ \\
\hline Smoking & $11(36 \%)$ & $8(27 \%)$ \\
\hline Pulmonary diseases (asthma, COPD) & $7(23 \%)$ & $8(27 \%)$ \\
\hline BMI $\left(\mathrm{kg} / \mathrm{m}^{2}\right)$ & $29.3 \pm 0.9$ & $30.4 \pm 1.3$ \\
\hline Target heart rate $(>70 / \mathrm{min})$ & $23(76.7 \%)$ & $20(66.7 \%)$ \\
\hline Atrial fibrillation & $7(23 \%)$ & $10(33.3 \%)$ \\
\hline \multicolumn{3}{|l|}{ Concomitant treatment } \\
\hline ACE inhibitor/ARB & $28(93 \%)$ & $29(97 \%)$ \\
\hline Beta-blocker & $29(97 \%)$ & $28(93 \%)$ \\
\hline MRA & $23(76.7 \%)$ & $21(70 \%)$ \\
\hline Ivabradine & $6(20 \%)$ & $6(20 \%)$ \\
\hline \multicolumn{3}{|l|}{ Diuretics } \\
\hline Loop diuretics (furosemide, etacrynic acid) & $27(90 \%)$ & $28(93 \%)$ \\
\hline Thiazide or thiazide-like diuretics (hypothiazide, indapamide, etc.) & $8(27 \%)$ & $9(30 \%)$ \\
\hline \multicolumn{3}{|l|}{ Device therapy } \\
\hline CRT-P/D & $9(30 \%)$ & $7(23.2 \%)$ \\
\hline ICD & $4(13 \%)$ & $3(10 \%)$ \\
\hline
\end{tabular}

Baseline characteristics of the study population according to the treatment arms. Values are expressed as mean \pm SEM. There were no significant differences in characteristics between RES- (resveratrol-) and placebo-treated groups at baseline. ACEI: angiotensin-converting enzyme inhibitors; ARB: angiotensin receptor blocker; BMI: body mass index; COPD: chronic obstructive pulmonary disease; CRT-P/D: cardiac resynchronization therapy-pacemaker/defibrillator; Dias. BP: diastolic blood pressure; ICD: implantable cardioverter-defibrillator; MRA: mineralocorticoid receptor antagonist; NT-proBNP: N-terminal prohormone of brain natriuretic peptide; Sys. BP: systolic blood pressure.

by paired two-sample Student's $t$-test. Differences between the groups were calculated by one-way analysis of variance (ANOVA) test. Data are expressed as mean \pm SEM (standard error of mean). Significance level was defined as $p<0.05$. The homogeneity of the groups was tested by Levene's $F$ -test. The impact of parameters on 6MWT results was analyzed by the Pearson correlation analysis.

\section{Results}

3.1. Hemorheological Parameters. The apparent WBV was increased $(p<0.05)$ in heart failure patients, but Hct and
PV did not show any difference in either resveratrol or placebo group compared to the control group at baseline. According to our results, resveratrol had no effect on Hct, $\mathrm{PV}$, or WBV and no difference was observed between the two groups (RES and placebo) either at baseline or after the 3-month follow-up period (Table 2).

The AI and the threshold shear rate $(\gamma)$ were significantly higher, and aggregation half time $\left(t_{1 / 2}\right)$ was significantly lower in both the resveratrol and placebo groups compared to the control group at baseline $(p<0.05)$. The $M_{1}$ measured by Myrenne $(p<0.05)$, the LORCA AI $(p<0.05)$, and LORCA $\gamma$ $(p<0.05)$ decreased significantly after RES treatment. 
TABLE 2: Effect of resveratrol on hemorheological parameters.

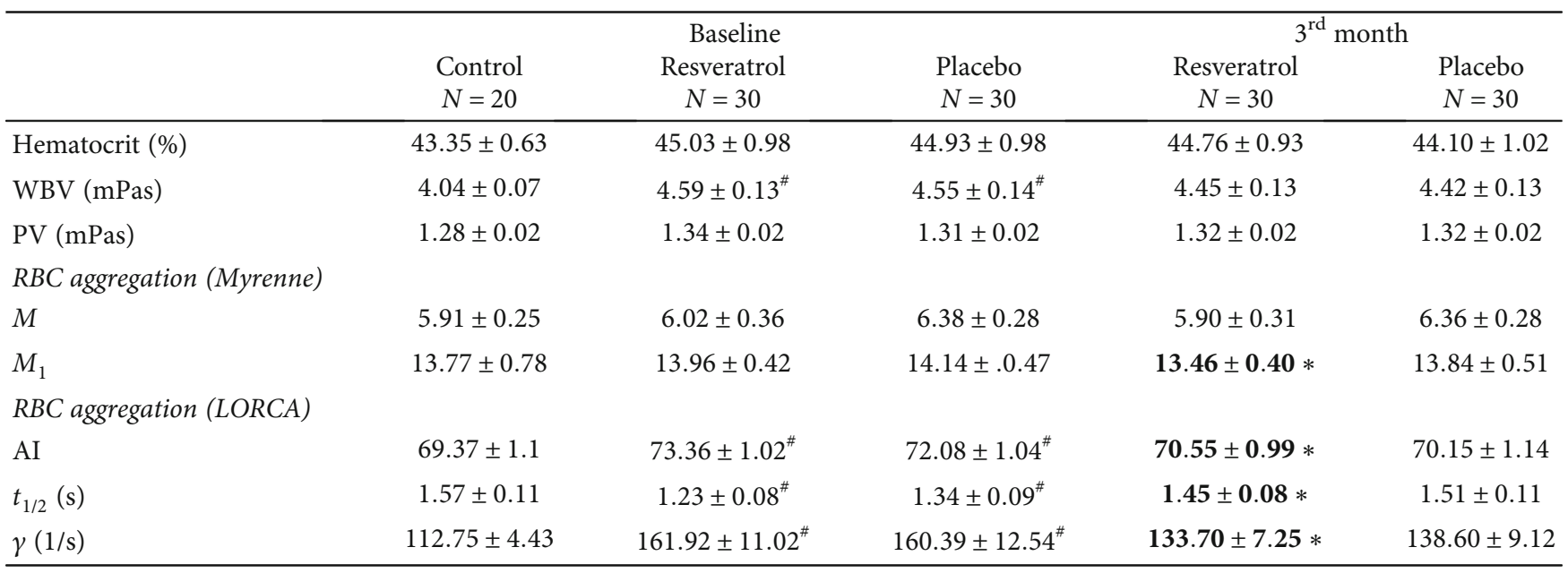

Values are expressed as mean \pm SEM. " Significant difference in the resveratrol or placebo groups compared to the control group at baseline; $*$ significant difference in $3^{\text {rd }}$ month values of the resveratrol group compared to the baseline values of the resveratrol group $(p<0.05)$. Baseline: measured values at randomization in the resveratrol group or in the placebo group; $3^{\text {rd }}$ month: patients treated with resveratrol or placebo for 3 months; AI: aggregation index; $t_{1 / 2}$ (s): aggregation half time; $M$ and $M_{1}$ values: aggregation indices at different rotation speeds of the aggregometer; PV: plasma viscosity; RBC: red blood cell; WBV: whole blood viscosity; $\gamma(1 / \mathrm{s})$ : threshold shear rate.
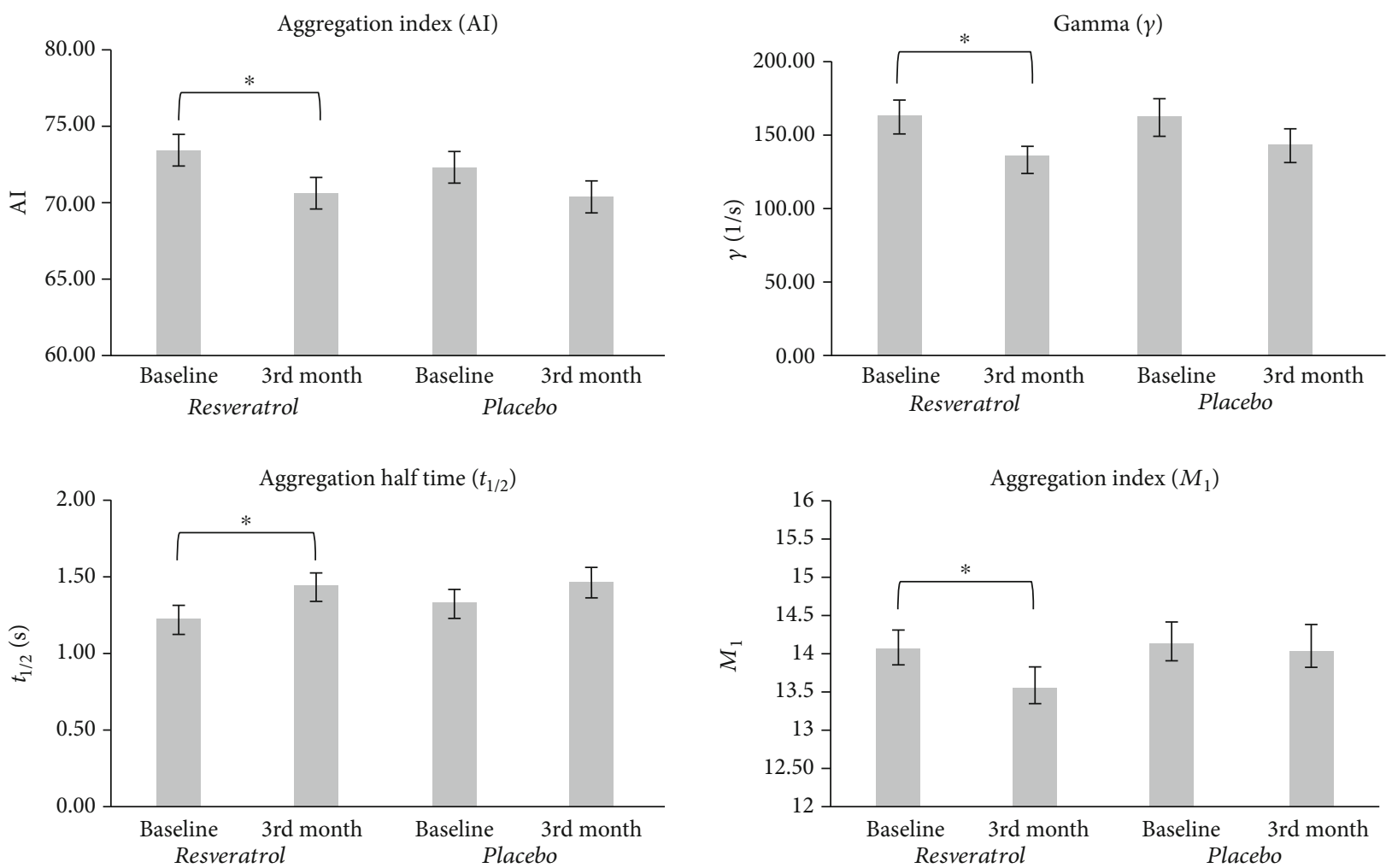

FIGURE 2: Effect of resveratrol on red blood cell aggregation. Values are expressed as mean \pm SEM. Star $(*)=$ significant difference in $3^{\text {rd }}$ month values of the resveratrol group compared to the baseline values of the resveratrol group $(p<0.05)$. Baseline: measured values at randomization in the resveratrol or placebo group; $3^{\text {rd }}$ month: patients treated with resveratrol or placebo for 3 months; $M_{1}$ value: aggregation index at $3 \mathrm{~s}^{-1}$ rotation speed of the Myrenne aggregometer; AI: aggregation index measured by LORCA aggregometer; $t_{1 / 2}(\mathrm{~s})$ : aggregation half time; $\gamma(1 / \mathrm{s})$ : threshold shear rate. 
TABLE 3: Effect of resveratrol on red blood cell deformability.

\begin{tabular}{lcccr}
\hline & Resveratrol (EI) & Baseline & Resveratrol (EI) & 3rd month \\
& & Placebo (EI) & & \\
Shear stress & & & & \\
$30(\mathrm{~Pa})$ & $0.619 \pm 0.003$ & $0.623 \pm 0.001$ & $0.621 \pm 0.002$ & $0.623 \pm 0.001$ \\
$3(\mathrm{~Pa})$ & $0.415 \pm 0.004$ & $0.420 \pm 0.003$ & $0.414 \pm 0.004$ & $0.422 \pm 0.003$ \\
$0.3(\mathrm{~Pa})$ & $0.008 \pm 0.009$ & $0.009 \pm 0.006$ & $0.005 \pm 0.007$ & $0.009 \pm 0.006$ \\
\hline
\end{tabular}

Values are expressed as mean \pm SEM. There was no difference in the EI values at any shear stresses between the placebo, resveratrol baseline, and $3^{\text {rd }}$ month groups. Baseline: measured values at randomization in the resveratrol or placebo group; $3^{\text {rd }}$ month: patients treated with resveratrol or placebo for 3 months. EI: elongation index; Pa: pascal.

Furthermore, $t_{1 / 2}$ measured by LORCA also demonstrated a significant $(p<0.05)$ alteration after 3 months compared to baseline (Table 2, Figure 2).

The deformability of RBCs at any measured shear stresses did not show significant changes in either group after 3 months compared to baseline (Table 3 ), though an increasing tendency of EI at high shear stress could be seen in the resveratrol group.

3.2. Six-Minute Walk Test. The 6-minute walk distance improved significantly $(275 \pm 19.3 \mathrm{~m}$ vs. $298 \pm 21.6 \mathrm{~m}, p<$ $0.05)$ after RES treatment compared to baseline but did not change in the placebo group (NS).

3.3. Relationship between 6-Minute Walk Distance and Hemorheological Variables. Hematocrit $(r=0.343, p<0.05)$ and whole blood viscosity $(r=0.308, p<0.05)$ had a moderate positive correlation with the $6 \mathrm{MWT}$ (correlation indices of the baseline pooled group). In the resveratrol group, significant correlation could be revealed between the 6-minute walk distance and the erythrocyte aggregation after 3 months: lower $M_{1}$ aggregation index $(r=-0.269, p<0.05)$ and lower $\gamma(-0.417, p<0.05)$ were associated with longer walk distance. In addition, in this subgroup, significant positive correlation was found between 6MWT and EI at high shear stresses (EI30: $r=0.480, p<0.005)$.

\section{Discussion}

The altered hemorheological factors in heart failure may play an important role in the complex impairment of microcirculation and the progression of heart failure. In previous studies, hemorheological parameters (WBV, plasma fibrinogen level, and RBC aggregation and deformability) were found significantly worse in heart failure patients than in healthy people [6]. Reduced peripheral blood flow, oxygen transport (hypoxemia), and increased oxidative stress in heart failure are described to cause RBC disorders [5, 6, 15]. Damage to red blood cells by ROS results in abnormalities in the function, morphology, and metabolism of erythrocyte including RBC aggregation and deformability [15, 31]. Cytoskeletal and membrane proteins and lipids are oxidized by ROS, which possibly increases the tendency of "damaged" erythrocytes to adhere with other erythrocytes thereby increasing RBC aggregation [32]. Our results demonstrated increased erythrocyte aggregation in heart failure patients compared to age-matched patients with moderate cardiovascular risk profile without heart failure (Table 2). Moreover, hypoxemia-induced rise in hematocrit (increased erythrocyte production) and elevated blood viscosity were observed in heart failure $[6,26]$, while in advanced stages of heart failure hematocrit level often decreases due to increased plasma volume (hemodilution), iron deficiency, and/or bone marrow depression caused by excessive cytokine and ROS production $[33,34]$.

According to literature, RES may have a protective role against the development of cardiovascular diseases. Primarily, RES is thought to be responsible for the cardioprotective effect of red wine (French paradox). The bioactive polyphenol resveratrol possesses antioxidant properties, reduces oxidative stress in animal models, and may contribute to the preservation of cardiac structure and function in animals [17-19]. Animal studies showed that RES can stabilize erythrocytes by the reduction of erythrocyte osmotic fragility [35]. RES was also described to maintain vascular endothelial function and to dilate blood vessels [19]. A previous human study of our working group assessing endothelial dysfunction detected significant improvement in vasorelaxation in REStreated patients [8]. Other authors reported that resveratrol can change the properties of plasma proteins and preserve the structure of fibrinogen from conformational alterations [36-38], which may influence RBC aggregation as well.

The effects of resveratrol on hemorheological parameters have already been evaluated in some animal and human trials; however, they have not been studied in heart failure $[8,24]$.

The main findings of our present trial after a 3-month follow-up period are as follows.

(1) RBC aggregation was decreased significantly in patients treated with resveratrol; (2) macrorheological parameters did not change significantly; (3) the 6-minute walk distance was increased significantly in resveratrol-treated patients; (4) relationship was detected between the 6-minute walk distance and some hemorheological variables (erythrocyte aggregation, erythrocyte deformability, hematocrit, and whole blood viscosity).

We found no significant change in $H c t, W B V$, and $P V$ values after the 3-month RES treatment. Similar results were seen in a previous study of our workgroup [8].

On the other hand, we could demonstrate a significant decrease of $R B C$ aggregation in our patients after RES treatment via several parameters. Based on our results-which 
confirm a previous study [6] $-\mathrm{RBC}$ aggregation is deteriorated significantly in patients with heart failure, while in this study RES treatment partially reversed these changes of RBC aggregation. Though some difference could be seen between the results of LORCA and Myrenne, these are probably the consequences of the different principles of operation and precision of the instruments [25].

The decrease in aggregation may be a consequence of the antioxidant properties of RES and the modifications of plasma proteins as well. According to one of the accepted theories, RBC aggregation is due to the bridging between adjacent cells by specific plasma proteins (e.g., fibrinogen, large molecular weight globulins) and influenced by the concentration and specific binding to the erythrocyte membrane of fibrinogen. It is known that polyphenols are bound to plasma proteins due to their poor water solubility. RES may change the properties of plasma proteins and RBC surface molecules, thus reducing the ability to form cross-links between cellular components and decreasing erythrocyte aggregation $[23,37,38]$. The reduction of RBC aggregation may have a positive effect on the flow properties of coronary microcirculation, which can be especially important in heart failure.

In this trial, we could not demonstrate the change of $R B C$ deformability after RES treatment by any ektacytometry parameters.

$6 M W T$ is a routine diagnostic procedure to quantify the exercise capacity of heart failure patients [39]. In this double-blind, randomized placebo-controlled study, resveratrol-treated patients had longer walk distance after the 3-month follow-up period, which is concordant with the results of a previous study [40]. Furthermore, a positive correlation was found between the functional capacity and the favorable hemorheological alterations as well. The improving hemorheological parameters may partly contribute to the longer walk distance in patients treated with resveratrol.

\section{Conclusion}

In our in vivo human study, we confirmed the beneficial effect of RES on erythrocyte aggregation in heart failure. The decrease of RBC aggregation by RES may positively influence the microcirculation, tissue perfusion, and oxygen supply, which may contribute to the improvement of the coronary and peripheral blood flow and probably increase the exercise capacity of patients with heart failure with reduced ejection fraction.

5.1. Limitations of the Study. This study has potential limitations. Although the study was double-blind, randomized, (1) the number of patients enrolled was low and the (2) follow-up period was relatively short. The rheological changes observed were significant (3) but the degree of change was modest.

Several positive effects of resveratrol by different mechanisms are proven. (4) It is not known exactly whether the positive effect of resveratrol on cardiac function and exercise capacity is due to these rheological changes or other mecha- nisms are also responsible for it. Further studies are required on heart failure patients to properly understand the exact biochemical and cellular mechanism of resveratrol.

\section{Data Availability}

The individual patient data used to support the findings of this study are available from the corresponding author upon request.

\section{Conflicts of Interest}

All authors have approved the manuscript for submission, and we have no conflicts of interest to disclose.

\section{Authors' Contributions}

All authors made a substantial contribution to the concept and design, acquisition of data, or analysis and interpretation of the data. All authors also assisted in drafting the article or revising it critically for important intellectual content.

\section{Acknowledgments}

This study was supported by an Economic Development and Innovation Operative Programme Grant (GINOP 2.3.2-152016-00048) and by an Institutional Developments for Enhancing Intelligent Specialization Grant (EFOP-3.6.2-162017-0006) and Establishing Thematic Scientific and Cooperation Network for Clinical Research (EFOP-3.6.2-162017-0009) from the Hungarian National Research, Development and Innovation Office.

\section{References}

[1] A. L. Bui, T. B. Horwich, and G. C. Fonarow, "Epidemiology and risk profile of heart failure," Nature Reviews Cardiology, vol. 8, no. 1, pp. 30-41, 2011.

[2] P. Ponikowski, A. A. Voors, S. D. Anker et al., "2016 ESC guidelines for the diagnosis and treatment of acute and chronic heart failure," European Heart Journal, vol. 37, no. 27, pp. 2129-2200, 2016.

[3] E. Tanai and S. Frantz, "Pathophysiology of heart failure," Comprehensive Physiology, vol. 6, no. 1, pp. 187-214, 2015.

[4] H. H. Lipowsky, "Microvascular rheology and hemodynamics," Microcirculation, vol. 12, no. 1, pp. 5-15, 2005.

[5] K. Matschke and F. Jung, "Regulation of the myocardial microcirculation," Clinical Hemorheology and Microcirculation, vol. 39, no. 1-4, pp. 265-279, 2008.

[6] I. Tikhomirova, E. Petrochenko, A. Muravyov et al., "Microcirculation and blood rheology abnormalities in chronic heart failure," Clinical Hemorheology and Microcirculation, vol. 65, no. 4, pp. 383-391, 2017.

[7] K. Biro, B. Sandor, D. Kovacs et al., "Lower limb ischemia and microrheological alterations in patients with diabetic retinopathy," Clinical Hemorheology and Microcirculation, vol. 69, no. 1-2, pp. 23-35, 2018.

[8] K. Magyar, R. Halmosi, A. Palfi et al., "Cardioprotection by resveratrol: a human clinical trial in patients with stable coronary artery disease," Clinical Hemorheology and Microcirculation, vol. 50, no. 3, pp. 179-187, 2012. 
[9] A. S. Popel and P. C. Johnson, "Microcirculation and hemorheology," Annual Review of Fluid Mechanics, vol. 37, no. 1, pp. 43-69, 2005.

[10] J. A. Dormandy and G. Nash, "Importance of red cell aggregation in venous pathology," Clinical Hemorheology and Microcirculation, vol. 7, pp. 119-122, 2016.

[11] T. Urdulashvili, N. Momtselidze, M. Mantskava, N. Narsia, and G. Mchedlishvili, "Hemorheological, microvascular and hemodynamic disorders during coronary heart disease," Georgian Medical News, vol. 136, pp. 55-57, 2016.

[12] P. Aukrust, T. Ueland, E. Lien et al., "Cytokine network in congestive heart failure secondary to ischemic or idiopathic dilated cardiomyopathy," The American Journal of Cardiology, vol. 83, no. 3, pp. 376-382, 1999.

[13] L. Gullestad, T. Ueland, L. E. Vinge, A. Finsen, A. Yndestad, and P. Aukrust, "Inflammatory cytokines in heart failure: mediators and markers," Cardiology, vol. 122, no. 1, pp. 2335,2012 .

[14] H. Tsutsui, S. Kinugawa, and S. Matsushima, "Oxidative stress and heart failure," American Journal of Physiology-Heart and Circulatory Physiology, vol. 301, pp. H2181-H2190, 2011.

[15] K. B. Pandey and S. I. Rizvi, "Biomarkers of oxidative stress in red blood cells," Biomedical Papers, vol. 155, no. 2, pp. 131136, 2011.

[16] G. B. Nash, R. B. Wenby, S. O. Sowemimo-Coker, and H. J. Meiselman, "Influence of cellular properties on red cell aggregation," Clinical Hemorheology and Microcirculation, vol. 7, pp. 93-108, 2016.

[17] S. Renaud and M. De Lorgeril, "Wine, alcohol, platelets, and the French paradox for coronary heart disease," The Lancet, vol. 339, no. 8808, pp. 1523-1526, 1992.

[18] A. Csiszar, "Anti-inflammatory effects of resveratrol: possible role in prevention of age-related cardiovascular disease," Annals of the New York Academy of Sciences, vol. 1215, no. 1, pp. 117-122, 2011.

[19] M. M. Sung and J. R. B. Dyck, "Therapeutic potential of resveratrol in heart failure," Annals of the New York Academy of Sciences, vol. 1348, no. 1, pp. 32-45, 2015.

[20] B. N. M. Zordoky, I. M. Robertson, and J. R. B. Dyck, "Preclinical and clinical evidence for the role of resveratrol in the treatment of cardiovascular diseases," Biochimica et Biophysica Acta (BBA) - Molecular Basis of Disease, vol. 1852, no. 6, pp. 1155-1177, 2015.

[21] M. Lagouge, C. Argmann, Z. Gerhart-Hines et al., "Resveratrol improves mitochondrial function and protects against metabolic disease by activating SIRT1 and PGC-1alpha," Cell, vol. 127, pp. 1109-1122, 2006, 6.

[22] X. S. Gu, Z. B. Wang, Z. Ye et al., "Resveratrol, an activator of SIRT1, upregulates AMPK and improves cardiac function in heart failure," Genetics and Molecular Research, vol. 13, no. 1, pp. 323-335, 2014.

[23] A. Toth, B. Sandor, J. Papp et al., "Moderate red wine consumption improves hemorheological parameters in healthy volunteers," Clinical Hemorheology and Microcirculation, vol. 56, no. 1, pp. 13-23, 2014.

[24] Y. Wang, H. Cui, F. Niu et al., "Effect of resveratrol on blood rheological properties in LPS-challenged rats," Frontiers in Physiology, vol. 9, p. 1202, 2018.

[25] O. K. Baskurt, M. Boynard, G. C. Cokelet et al., "New guidelines for hemorheological laboratory techniques," Clinical Hemorheology and Microcirculation, vol. 42, no. 2, pp. 75-97, 2009.
[26] K. Toth, G. Kesmarky, and T. Alexy, "Clinical significance of hemorheological alterations," in Handbook of Hemorheology and Hemodynamics, O. K. Baskurt, M. R. Hardeman, M. W. Rampling, and H. J. Meiselman, Eds., pp. 392-432, IOS Press, 2007.

[27] M. R. Hardeman, J. G. Dobbe, and C. Ince, "The laser-assisted optical rotational cell analyzer (LORCA) as red blood cell aggregometer," Clinical Hemorheology and Microcirculation, vol. 25, pp. 1-11, 2011.

[28] H. J. Klose, E. Volger, H. Brechtelsbauer, L. Heinich, and H. Schmid-Schonbein, "Microrheology and light transmission of blood," Pflügers Archiv European Journal of Physiology, vol. 333, no. 2, pp. 126-139, 1972.

[29] M. R. Hardeman, P. T. Goedhart, and N. H. Schut, "Laserassisted optical rotational cell analyser (L.O.R.C.A.); II. red blood cell deformability: elongation index versus cell transit time," Clinical Hemorheology and Microcirculation, vol. 14, no. 4, pp. 619-630, 1994.

[30] R. O. Crapo, R. Casaburi, A. L. Coates et al., "ATS statement: guidelines for the six-minute walk test," American Journal of Respiratory and Critical Care Medicine, vol. 166, no. 1, pp. 111-117, 2002.

[31] M. Becatti, R. Marcucci, A. M. Gori et al., "Erythrocyte oxidative stress is associated with cell deformability in patients with retinal vein occlusion," Journal of Thrombosis and Haemostasis, vol. 14, no. 11, pp. 2287-2297, 2016.

[32] P. Gyawali and R. S. Richards, "Association of altered hemorheology with oxidative stress and inflammation in metabolic syndrome," Redox Report, vol. 20, no. 3, pp. 139-144, 2014.

[33] A.-S. Androne, S. D. Katz, L. Lund et al., "Hemodilution is common in patients with advanced heart failure," Circulation, vol. 107, no. 2, pp. 226-229, 2003.

[34] P. O. Iversen, P. R. Woldbaek, T. Tønnessen, and G. Christensen, "Decreased hematopoiesis in bone marrow of mice with congestive heart failure," American Journal of Physiology-Regulatory, Integrative and Comparative Physiology, vol. 282, no. 1, pp. R166-R172, 2002.

[35] Y. Meng, M. Zhang, J. Xu, X.-M. Liu, and Q.-Y. Ma, "Effect of resveratrol on microcirculation disorder and lung injury following severe acute pancreatitis in rats," World Journal of Gastroenterology, vol. 11, no. 3, pp. 433-435, 2005.

[36] C. Bonechi, S. Lamponi, A. Donati et al., "Effect of resveratrol on platelet aggregation by fibrinogen protection," Biophysical Chemistry, vol. 222, pp. 41-48, 2017.

[37] D. Lominadze and W. L. Dean, "Involvement of fibrinogen specific binding in erythrocyte aggregation," FEBS Letters, vol. 517, no. 1-3, pp. 41-44, 2002.

[38] X. Weng, G. Cloutier, R. Beaulieu, and G. O. Roederer, "Influence of acute-phase proteins on erythrocyte aggregation," The American Journal of Physiology, vol. 271, 6 Part 2, pp. H2346H2352, 1996.

[39] P. Raj, X. Lieben Louis, S. J. Thandapilly, A. Movahed, S. Zieroth, and T. Netticadan, "Potential of resveratrol in the treatment of heart failure," Life Sciences, vol. 95, no. 2, pp. 63-71, 2014.

[40] L. Gliemann, J. F. Schmidt, J. Olesen et al., "Resveratrol blunts the positive effects of exercise training on cardiovascular health in aged men," The Journal of Physiology, vol. 591, no. 20 , pp. 5047-5059, 2013. 